\title{
Determination of Bulk Modulus for a Colloidal Crystal with Highly Charged Particles by DC Electric Field
}

\author{
Linwei Wang, ${ }^{\dagger}$ Shenghua $\mathrm{Xu},{ }^{*},{ }^{\dagger},{ }^{\ddagger} \odot$ Hongwei Zhou, ${ }^{\dagger}$ Zhiwei Sun, ${ }^{\dagger}$ Wenze Ouyang, ${ }^{\dagger}$ \\ and Shenwei Wang ${ }^{\dagger,+}$ \\ ${ }^{\dagger}$ Key Laboratory of Microgravity, Institute of Mechanics, Chinese Academy of Sciences, Beijing 100190, China \\ ${ }^{*}$ School of Engineering Science, University of Chinese Academy of Sciences, Beijing 100049, China
}

ABSTRACT: In this study, bulk modulus of a colloidal crystal formed by highly charged particles is experimentally determined by applying direct current electric field. A theoretical expression is also proposed to independently predict the bulk modulus based on van't Hoff's law of osmotic pressure and the theory of Ohshima. The experimental result thus obtained agrees well with the theoretical expectation. In addition, results from both above-mentioned methods coincide with that inferred from the static structure factor.



\section{INTRODUCTION}

Highly charged particles in an aqueous environment can be self-assembled into a colloidal crystal with interparticle separation distances being significantly greater than the individual particle diameter. ${ }^{1-5}$ The elasticity of the colloidal crystal is one of its important research topics with theoretical and practical significance. ${ }^{6-8}$ However, unlike ordinary materials, elastic moduli of such kind of colloidal crystals are extraordinarily small (about 8 orders lower than those of metals), ${ }^{9,10}$ so the conventional measuring methods applicable to regular solids have become no longer valid. ${ }^{10,11}$

There have been some reports about investigation on Young's modulus of a colloidal crystal. Crandall and Williams ${ }^{12}$ once proposed a sedimentation equilibrium method for the determination of Young's modulus of the colloidal crystal with polystyrene particles by observing the variation of nearest interparticle distance with respect to the height of the colloidal crystal remaining at the sedimentation equilibrium between the gravity and repulsive interactions of particles. By the same method, Okubo ${ }^{6,13}$ obtained Young's modulus of the colloidal crystal with charged silica particles subsequently. Afterward, based on the studies of Tomita et al., ${ }^{14,15}$ Okubo $^{16}$ proposed the electrostatic field method by improving the above sedimentation equilibrium method through the substitution of gravitational field with the external direct current (DC) electric field.

We doubt the rationality of the above-mentioned sedimentation equilibrium and electrostatic field methods for measuring Young's modulus. Both of them imply that the colloidal crystal under unidirectional compression would expand freely in directions perpendicular to the direction of compression, just as the ordinary solid materials behave. However, in fact, it is not the case. The colloidal crystal has no fixed shape of its own and takes the shape of the container that holds it. Due to the constraint of container walls, the crystal is unable to expand in other directions under the unidirectional compression. Thus, in our opinion, these two methods are not suitable for the measurement of Young's modulus by neglecting the constraint of the container.

Although the above methods cannot be used to measure Young's modulus, either gravitational or electric field does provide a simple and effective way to load stress to the colloidal crystal. When the applied DC electric field is very low, the crystal structure is maintained and the colloidal particles do not migrate. Gravity loading is simple, but it needs quite a long time for the sedimentation process; also, the gravity is usually too weak to induce measurable deformation of the colloidal crystal, while the electric field intensity can be controlled to produce enough deformation. By the way, it should be noted that the voltage gradient along the direction of electric field is not homogeneous throughout when exerting a DC electric field on the colloidal suspension so that the electric field strength cannot be deduced directly from the incremental voltage per unit length between electrodes.

As for the determination of bulk modulus for charged colloidal suspension, a couple of methods have been proposed. The bulk modulus can be measured directly by osmotic stress. ${ }^{17}$ Static structure factor is a characterization of the scattering ability of materials to radiation that reflects average information of the structure of colloidal suspension, and therefore, it has also been used to evaluate the bulk modulus. ${ }^{18-24}$ The static structure factor can be obtained by small-angle X-ray scattering, static light scattering, and turbidimetry. Gapinski et al. ${ }^{19,20}$ proposed that the static

Received: May 7, 2019

Revised: August 14, 2019

Published: August 14, 2019 
structure factor can also be achieved from relevant parameters of the colloidal system. More recently, Junio et al. ${ }^{18}$ employed the optical bottle technique to measure the osmotic bulk modulus of colloidal suspension and compared their results with those achieved through a zero-wavenumber static structure factor.

In this paper, we present an experimental scheme for measuring the bulk modulus in which the osmotic stress is induced by an electrostatic field and state why this method is suitable for measuring the bulk modulus rather than Young's modulus. The experimental equipment, procedure, and the relevant theoretical basis are described. On the other hand, a theoretical expression to predict the bulk modulus directly is proposed in terms of interactions of counterions in the gap between particles. The calculation of bulk modulus using the structure factor is also performed for comparison. Results from the above three different approaches, including our experiment using a DC electric field method, theoretical prediction analyzing the interaction of counterions, and the previously reported calculation by the aid of the structure factor, are entirely consistent with each other.

\section{THEORY}

Young's Modulus or Bulk Modulus? The molecules in a standard crystal are tightly bound together and can only vibrate without moving. ${ }^{25}$ In contrast, particles in the colloidal crystal are bound rather loosely and hence can easily move around like molecules in a liquid; then, the loading stress to the colloidal crystal can be transferred in all directions equally because of the fluidity of constituent particles. ${ }^{10}$ Furthermore, the colloidal crystal itself grows under the hydrostatic pressure of liquid solvent. ${ }^{26}$ Thus, at any point of the colloidal crystal at rest (static state), the normal stress in all directions equals with each other, i.e., $\sigma_{x x}=\sigma_{y y}=\sigma_{z z}$, where $x, y$, and $z$ are axes of a Cartesian coordinate system and $x$ is on the direction of gravitational or electrostatic field. Since the normal stress in all directions shares the same value $(-p)$, the three mutually perpendicular normal strains $\varepsilon_{x x}, \varepsilon_{y y}$, and $\varepsilon_{z z}$ should also have the same value at one point of the colloidal crystal.

For isotropic materials, we have the equation of Hooke's $\operatorname{law}^{27,28}$ as

$$
\varepsilon_{x x}=\frac{1}{E_{\mathrm{Y}}}\left[\left(\sigma_{x x}-\nu\left(\sigma_{y y}+\sigma_{z z}\right)\right]\right.
$$

where $E_{\mathrm{Y}}$ is Young's modulus and $\nu$ is Poisson's ratio. For colloidal crystal with $\sigma_{x x}$ loaded by the sedimentation equilibrium or electrostatic field method, $\nu$ is unknown and $\sigma_{y y}$ and $\sigma_{z z}$ are definitely nonzero due to constraints of container walls. Consequently, the Young's modulus, $E_{\mathrm{Y}}$, cannot be obtained as expected by the sedimentation equilibrium or electrostatic field method, ${ }^{6,12,13,16}$ although $\varepsilon_{x x}$ and $\sigma_{x x}$ can be acquired by experiments.

Since the strains have the same value in all directions at a point of the colloidal crystal, stresses induced by gravity or electrostatic field will result in isotropic compression of colloidal crystals. The reciprocal of the compressibility then gives the bulk modulus of the colloidal crystal, which can be obtained by analysis of regression from experimental results through the formula

$$
K=-\frac{\mathrm{d} p}{\mathrm{~d} \varepsilon_{\mathrm{b}}}
$$

where $\varepsilon_{\mathrm{b}}=\varepsilon_{x x}+\varepsilon_{y y}+\varepsilon_{z z}$.

Overall, such a stress loading method referred in the papers of Crandall et al. ${ }^{12}$ and Okubo ${ }^{6,13,16}$ cannot be directly used to measure Young's modulus but rather can be used to measure the bulk modulus.

Theoretical Formulation for Measuring Bulk Modulus by the Electric Field Method. In this subsection, we will demonstrate how to measure the bulk modulus of the colloidal crystal by applying electric field between parallel plate electrodes.

Although in some respect the colloidal system in a weak DC electric field is similar to that in a gravitational field, it is not straightforward to evaluate the relevant force on colloidal particles. As known, the charged particle suspended in liquid medium are surrounded by a diffuse layer of counterions according to the theory of electric double layer. When the DC electric field is applied, not only the electric force but also the forces contributed by counterions will be exerted on the particle. Therefore, it becomes complicate to calculate the forces applied to the particle directly. As a simplified approach to analyze the total forces caused by the DC electric field, following the procedure proposed by researchers before, ${ }^{29-31}$ the model of spherical Wigner-Seitz cell is adopted in this study.

According to the model of a spherical Wigner-Seitz cell $^{29,32,33}$ as shown in Figure 1, each particle with radius $a$

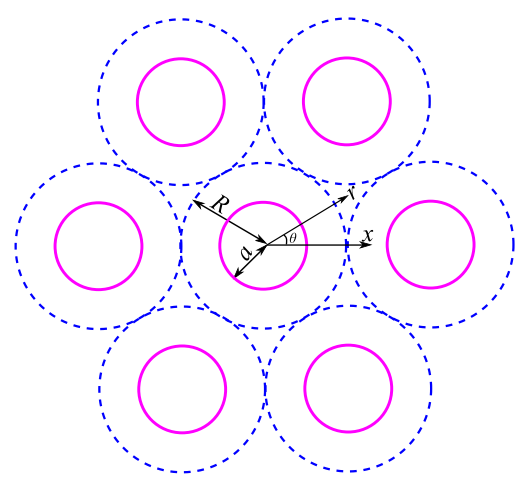

Figure 1. Schematic of spherical Wigner-Seitz cells (solid circles represent colloidal particles with radius $a$; dashed circles represent spherical Wigner-Seitz cells with radius $R$ ).

is embedded into a concentric virtual sphere with radius $R$ satisfying the equation

$$
\phi=\left(\frac{a}{R}\right)^{3}
$$

where $\phi$ is the particle volume fraction of the colloidal suspension. The virtual sphere is referred to as a spherical Wigner-Seitz cell. Hence, the entire volume of suspension can be regarded to be occupied by such spherical Wigner-Seitz cells and each spherical cell appears to be electrically neutral.

Since negative charged particles were used in this study, the direction of force field acting on particles is opposite to that of the electric field. As discussed above, the electrostatic field will make the volume of a Wigner-Seitz cell at one end smaller than that at another end. The volume expansion, $\varepsilon_{\mathrm{b}}$, can also be defined as

$$
\varepsilon_{\mathrm{b}}=\frac{\mathrm{d} V_{\text {cell }}}{V_{\text {cell }}}
$$


where $V_{\text {cell }}$ is the volume of a single spherical Wigner-Seitz cell as

$$
V_{\text {cell }}=\frac{4}{3} \pi R^{3}
$$

Thus, $K$ can also be written as

$$
K=-V_{\text {cell }} \frac{\mathrm{d} p}{\mathrm{~d} V_{\text {cell }}}
$$

When the colloidal crystal is at equilibrium under the application of external electric field, eq 6 can be rewritten as

$$
K=-V_{\text {cell }}^{(0)} \frac{\left.\frac{\mathrm{d} p(x)}{\mathrm{d} x}\right|_{d_{\exp }=d_{\text {exp }}^{(0)}}}{\left.\frac{\mathrm{d} V_{\text {cell }}(x)}{\mathrm{d} x}\right|_{d_{\exp }=d_{\exp }^{(0)}}}
$$

after setting up an $x$-axis with its origin at the middle point between two electrodes, as shown in Figure 2. Here, $V_{\text {cell }}^{(0)}$ is the original volume of a spherical Wigner-Seitz cell and $d_{\exp }^{(0)}$ is the original nearest interparticle distance when no external electric field is applied.

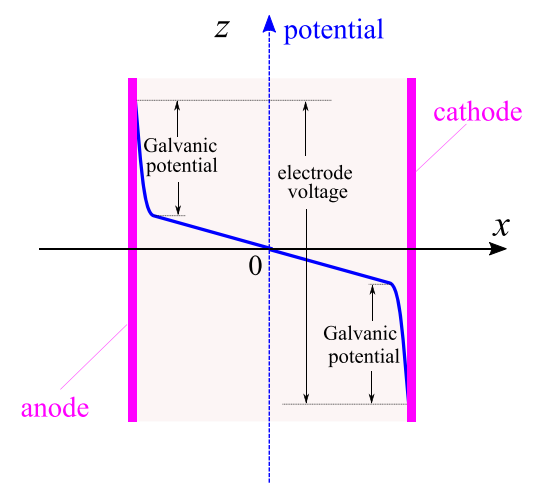

Figure 2. Schematic of $x$-axis and electric potential change inside colloidal suspension.

In this work, all of the formed colloidal crystals are bodycentered cubic (bcc) structures and then

$$
V_{\text {cell }}(x)=\frac{4 d_{\text {exp }}^{3}(x)}{3 \sqrt{3}}
$$

Thus,

$$
\left.\frac{\mathrm{d} V_{\text {cell }}(x)}{\mathrm{d} x}\right|_{d_{\text {exp }}=d_{\text {exp }}^{(0)}}=\left.\frac{4}{\sqrt{3}}\left(d_{\exp }^{(0)}\right)^{2} \frac{\mathrm{d} d_{\text {exp }}(x)}{\mathrm{d} x}\right|_{d_{\text {exp }}=d_{\text {exp }}^{(0)}}
$$

Because the strength of the applied electric filed is extremely small, we can assume that the values of $\mathrm{d} d_{\exp }(x) / \mathrm{d} x$ and $\mathrm{d} p(x)$ / $\mathrm{d} x$ are constant in the bulk of suspension; then, there is

$$
\left.\frac{\mathrm{d} p(x)}{\mathrm{d} x}\right|_{d_{\exp }=d_{\text {exp }}^{(0)}}=\frac{\mathrm{d} p(x)}{\mathrm{d} x}
$$

and eq 9 can be rewritten as

$$
\left.\frac{\mathrm{d} V_{\text {cell }}(x)}{\mathrm{d} x}\right|_{d_{\text {exp }}=d_{\text {exp }}^{(0)}}=\frac{4}{\sqrt{3}}\left(d_{\exp }^{(0)}\right)^{2} \frac{\mathrm{d} d_{\exp }(x)}{\mathrm{d} x}
$$

To determine the directional derivative of pressure in the direction of $x$-axis, $\mathrm{d} p(x) / \mathrm{d} x$, the strength of the external electric field, $E$, applied to the colloidal suspension is needed to be known. However, there is Galvanic potential difference between the electrode and the bulk of suspension because the ions and charged particles can condense at surfaces of the electrodes to form double layers to induce non-Ohmic interfacial resistances there. ${ }^{34,35}$ The schematic of potential change is also shown in Figure 2. Hence, the actual strength of the external electric field that the suspension is subjected to is much smaller than $U_{\text {ele }} / L_{\text {ele }}$, where $U_{\text {ele }}$ is the voltage between electrodes and $L_{\text {ele }}$ is the spacing between electrodes. To determine the field strength in suspension, the differential form of Ohm's law ${ }^{36-38}$ is used in the scalar form

$$
j=\sigma_{0} E
$$

where $j$ is the magnitude of current density and $\sigma_{0}$ is the electric conductivity of the medium of suspension (counterions). This form of Ohm's law is especially for electric properties at the microlevel. Additionally, there are only one kind of counterions in the salt-free suspension, i.e., $\mathrm{H}^{+}$ions, which are dissociated from the surface function groups of particles, so $\sigma_{0}$ can be estimated by the formula ${ }^{36,39-41}$

$$
\sigma_{0}=\frac{\bar{n}_{\mathrm{H}^{+}} \Lambda_{\mathrm{H}^{+}}}{N_{\mathrm{A}}}
$$

where $\bar{n}_{\mathrm{H}^{+}}$is the average number density of counterions described as

$$
\bar{n}_{\mathrm{H}^{+}}=\frac{3 Z^{*}}{4 \pi R^{3}}
$$

Here, $\Lambda_{\mathrm{H}^{+}}$is the limiting molar conductivity of $\mathrm{H}^{+}$ions and $\Lambda_{\mathrm{H}^{+}}=349.65 \times 10^{-4} \mathrm{~S} \mathrm{~m}^{2} \mathrm{~mol}^{-1}$ at the temperature of $25^{\circ} \mathrm{C}$, $\mathrm{Z}^{*}$ is the effective mobility charge number on the surface of a colloidal particle, and $N_{\mathrm{A}}$ is the Avogadro constant. Then, one can obtain the calculation formula of field strength applied to the suspension by combining eqs $3,12,13$, and 14 as

$$
E=\frac{4 \pi N_{\mathrm{A}} a^{3} j}{3 Z^{*} \phi \Lambda_{\mathrm{H}^{+}}}
$$

where the magnitude of current density, $j$, is calculated by

$$
j=\frac{I}{S_{\text {ele }}}
$$

Here, $I$ is the current intensity passed through the suspension and $S_{\text {ele }}$ is the contact surface area between an electrode and the suspension.

Subsequently, we estimate the internal stress inside the colloidal crystal based on the field strength, E. Since the particles used in this study have high surface charge, we can estimate the internal stress according to theoretical studies of Ohshima, ${ }^{42,43}$ in which the effective mobility charge, $Z^{*}$, satisfies the condition

$$
\frac{Z^{*} l_{\mathrm{B}}}{a}>\ln \left(\frac{1}{\phi}\right)
$$

where $l_{\mathrm{B}}$ is the Bjerrum length

$$
l_{\mathrm{B}}=\frac{e^{2}}{4 \pi \epsilon_{0} \epsilon_{\mathrm{r}} k_{\mathrm{B}} T}
$$


$\epsilon_{0}$ is the dielectric permittivity of vacuum and $\epsilon_{\mathrm{r}}$ is the relative permittivity of water.

Since the Wigner-Seitz cell is electrically neutral, there is no net electric force on the unit cell. However, there is hydrodynamic force on the surface of the cell $F_{\mathrm{H}}$, which is related to the electric field. The resultant of hydrodynamic force, $F_{\mathrm{H}}$, on the Wigner-Seitz cell is the surface integral of the local hydrodynamic pressure, $p$. From the Navier-Stokes equation, the value of $p$ can be deduced, which is related to the local electric potential. If the DC electric field is not present, the electric potential on that surface is equal everywhere. However, with DC electric potential, there will be a potential change along the direction of electric field

$$
\delta \psi(\boldsymbol{r})=-\boldsymbol{E} \cdot \boldsymbol{r}
$$

which is

$$
\delta \psi(R, \theta)=-E R \cos \theta
$$

on the surface of the Wigner-Seitz cell. Here, $r$ is the position vector and $\theta$ is a coordinate of the spherical coordinate system $(r, \theta, \varphi)$ of which the origin is at the center of the particle and $\theta=0$ points to the direction of the DC electric field, $E$, as shown in Figure 1. Such a potential change will cause asymmetrical hydrodynamic pressure

$$
\delta p(\boldsymbol{r})=-e n_{\mathrm{H}+}(r) \delta \psi(\boldsymbol{r})
$$

which is

$$
\delta p(R, \theta)=-e n_{\mathrm{H}^{+}}(R) \delta \psi(R, \theta)=e n_{\mathrm{H}^{+}}(R) E R \cos \theta
$$

on the surface of the Wigner-Seitz cell. Here, $n_{\mathrm{H}^{+}}$is the number density of counterions $\left(\mathrm{H}^{+}\right)$. As a result, the hydrodynamic force, $F_{\mathrm{H}}$, on the Wigner-Seitz cell is the surface integral of the local hydrodynamic pressure as

$$
\begin{aligned}
& F_{\mathrm{H}}=-\oint_{S} \delta p(R, \theta) \cos \theta \mathrm{d} S \\
& =-2 \pi R^{2} \int_{0}^{2 \pi}\left[e n_{\mathrm{H}^{+}}(R) E R \cos \theta\right] \sin \theta \cos \theta \mathrm{d} \theta \\
& =-\frac{4}{3} \pi E R^{3} e n_{\mathrm{H}^{+}}(R)
\end{aligned}
$$

The ion density in suspension meets the Boltzmann-type distribution by supposing a reference place with zero electric potential where the number density of $\mathrm{H}^{+}$ions equals $\bar{n}_{\mathrm{H}^{+}}$, so the number density of $\mathrm{H}^{+}$ions at the outer boundary of spherical Wigner-Seitz cell is

$$
n_{\mathrm{H}^{+}}(R)=\bar{n}_{\mathrm{H}^{+}} \exp \left[-\frac{e \psi(R)}{k_{\mathrm{B}} T}\right]
$$

where $\psi(R)$ is the electric potential at the outer boundary of the spherical Wigner-Seitz cell, $k_{\mathrm{B}}$ is the Boltzmann constant, and $T$ is the absolute temperature of suspension. According to the study of Ohshima, ${ }^{42,43}$ in the case that the particles take high charge density at the surface (eq 17 is satisfied) and the particle volume fraction is much small than $1(\phi \ll 1)$, the electric potential on the outer boundary surface of the spherical Wigner-Seitz cell is

$$
\psi(R)=\frac{k_{\mathrm{B}} T}{e} \ln \left[\frac{Z^{*} l_{\mathrm{B}}}{a \ln (1 / \phi)}\right]
$$

By substituting eqs 14 and 25 into eq 24, one can get

$$
n_{\mathrm{H}^{+}}(R)=\frac{3 a \ln (1 / \phi)}{4 \pi l_{\mathrm{B}} R^{3}}=\frac{3 a \ln \left(R^{3} / a^{3}\right)}{4 \pi l_{\mathrm{B}} R^{3}}
$$

Then, by substituting eq 26 into eq 23, the hydrodynamic force, $F_{\mathrm{H}}$, on the Wigner-Seitz cell can also be expressed as

$$
F_{\mathrm{H}}=-e E \frac{a}{l_{\mathrm{B}}} \ln \left(\frac{1}{\phi}\right)
$$

The minus sign at the right side of the last equation implicates that the direction of hydrodynamic force on the negatively charged particle is opposite to that of the DC electric field.

In addition, we have

$$
\frac{\mathrm{d} p(x)}{\mathrm{d} x}=\frac{F_{\mathrm{H}}}{V_{\mathrm{cell}}^{(0)}}
$$

in the bulk of suspension according to the theory of hydrostatics. $^{44}$ By substituting eqs $10,11,27$, and 28 to 7 , we have

$$
K=\frac{\frac{\sqrt{3} e E \phi^{2 / 3} \ln (1 / \phi)}{4 \sqrt[3]{3 \pi^{2}} a l_{\mathrm{B}}}}{\frac{\mathrm{d} d_{\text {exp }}(x)}{\mathrm{d} x}}
$$

which is applicable at the condition that eq 17 is satisfied.

Theoretical Model to Estimate the Bulk Modulus. In this subsection, we will derive an approximate analytic expression to estimate the osmotic bulk modulus of colloidal crystal formed by charged particles by means of combining the theory of Ohshima on the surface charge density-surface potential relationship ${ }^{42,43}$ for a particle in salt-free suspension with van't Hoff's law of osmotic pressure. ${ }^{4,10,45}$

The combination of the particles and counterions inside a spherical Wigner-Seitz cell is overall charged neutral. The repulsive force between particles is not due to the direct effect of electrostatics but the excess osmotic pressure of the counterions in the gap between colloidal particles. ${ }^{45}$ According to the van't Hoff's law of osmotic pressure, ${ }^{29,46,47}$ the osmotic pressure of counterions, $\Pi$, is expressed as

$$
\Pi=k_{\mathrm{B}} T n_{\mathrm{H}^{+}}(R)
$$

Accordingly, the bulk modulus of colloidal crystal can be defined as

$$
K=-V_{\text {cell }} \frac{\mathrm{d} \Pi}{\mathrm{d} V_{\text {cell }}}
$$

where $V_{\text {cell }}$ is the volume of a spherical Wigner-Seitz cell

$$
V_{\text {cell }}=\frac{4}{3} \pi R^{3}
$$

By substituting eqs 30 and 32 into eq 31, one can obtain

$$
K=-\frac{1}{3} k_{\mathrm{B}} T R \frac{\partial n_{\mathrm{H}^{+}}(R)}{\partial R}
$$

Now, by substituting eq 26 into eq 33, we have the theoretical expression to calculate the bulk modulus of the colloidal crystal assembled from highly charged particles in the salt-free suspension as

$$
K=\frac{3 k_{\mathrm{B}} T \phi[\ln (1 / \phi)-1]}{4 \pi l_{\mathrm{B}} a^{2}}
$$




\section{EXPERIMENTAL SECTION}

Sample Preparation. Two kinds of polystyrene particles (PS-1 and PS-2) carrying a large number of ionizable sulfate groups are used in this work. Their characteristic parameters are shown in Table 1 . Here, the effective mobility charge, $Z^{*}$, is

Table 1. Parameters of Colloidal Particles

$\begin{array}{ccccc} & \text { diameter } & \text { polydispersity } & \begin{array}{c}\text { effective mobility } \\ \text { charge }\end{array} & \begin{array}{c}\text { effective elasticity } \\ \text { charge }\end{array} \\ \text { particles } & 2 a / \mathrm{nm} & \text { PDI } & Z^{*} & Z^{* \prime} \\ \text { PS-1 } & 95 & 0.033 & 962 & 517 \\ \text { PS-2 } & 75 & 0.335 & 781 & 457\end{array}$

measured from the fitting of the conductivity versus number density curve, ${ }^{48,49}$ while the effective elasticity charge, $Z^{* \prime}$, is determined from the fitting of shear modulus versus number density curve. ${ }^{50,51}$ The difference of these two kinds of effective charges has been discussed thoroughly. ${ }^{52,53}$ In this paper, the effective mobility charge, $Z^{*}$, is used to calculate the conductivity of suspension when the crystal structure is formed in the experimental method since it is directly measured from conductivity. The effective elasticity charge, $Z^{* \prime}$, is used to calculate the zero-q limit static structure factors for inferring the bulk moduli since it is measured from mechanical properties and is related to particle interaction and crystal structure.

After the sample of colloidal suspension with a specified volume fraction of particles is prepared, the electrolytic ions inside the suspension were removed by ion-exchange resins (IRN-150, EMD Millipore Corporation, Germany) until the electrical conductivity of suspension was reduced to the minimum. ${ }^{54,55}$ After deionization, the suspension sample was enclosed into the quartz cell together with the platinum electrodes using the parafilm. Then, the colloidal particles of the suspension inside the quartz cell could self-assemble into the colloidal crystal that can appear iridescent color while keeping at the room temperature. ${ }^{55}$ It is worth noting that the enclosed sample cell should be immersed into an ultrasonic bath for some seconds before experiments to make sure the homogeneity and isotropy of the colloidal crystal.

Experimental Setup. The experimental setup designed to determine the bulk modulus of charged colloidal crystal comprises three units: a sample unit, a circuit unit, and an optical unit. The sample unit consists of the quartz cell and the colloidal suspension inside.

The circuit unit, of which the diagram is in Figure 3, consists of a pair of platinum electrodes, a DC power supply (HSPY300-01, Hanshengpuyuan, Beijing), a digit multimeter (Agilent 34401A 61/2, Keysight Technologies Inc.), and three resistors. This unit is used to exert stress by providing the electrostatic field and to measure the electric current through the suspension simultaneously. The details on how to deduce the exerted stress from the current intensity instead of the voltage between electrodes are given in the last section.

To be able to control the voltage between electrodes precisely, the resistors $\mathrm{R} 1$ and $\mathrm{R} 2$ are utilized to form a loop with the DC power supply to enlarge the needed output voltage of the power. Since the current intensity passed through the suspension between electrodes is on the order of $10^{-7} \mathrm{~A}$, which is quite small, another resistor R3 with a resistance of $10 \mathrm{k} \Omega$ is series-connected with the electrodes. Then, the total current intensity through the suspension can be

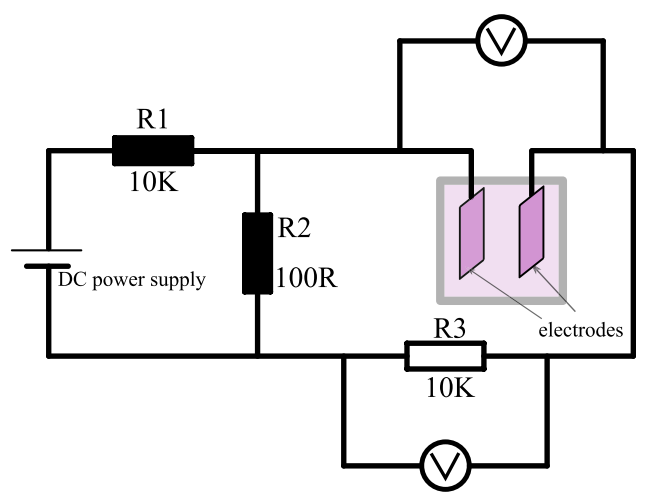

Figure 3. Circuit diagram.

obtained by measuring the voltage across R3. While measuring the voltages, the input impedance of the digit multimeter is shifted to $10 \mathrm{G} \Omega$ for the sake of high precision.

The optical unit, i.e., the reflection spectrometer including a tungsten halogen light source (AvaLight-HAL, Avantes Inc., the Netherlands), a fiber optic spectrometer (Avaspec-2048, Avantes Inc., the Netherlands), and a bifurcated fiber optic cable, is used to determine the shift of particles at different parts of the colloidal crystal by detecting the nearest interparticle distances. The diagram of this optical setup is shown in Figure 4. As shown in previous studies, ${ }^{54,56}$ the

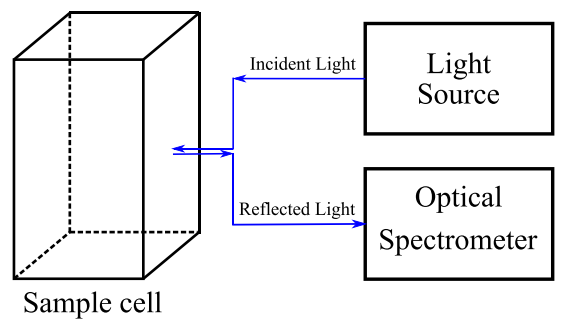

Figure 4. Diagram of optical setup.

nearest interparticle distances for both BCC and FCC structures can be calculated from the peak wavelength of the reflection curve through the formula

$$
d_{\exp }=0.6124 \lambda_{\mathrm{p}} / \nu_{\mathrm{w}}
$$

where $d_{\exp }$ is the nearest interparticle distance, $\lambda_{\mathrm{p}}$ is the wavelength at the peak of reflection curve, and $\nu_{\mathrm{w}}$ is the refractive index of water.

Here, we illustrate the general measurement procedure in our experiments by taking a PS-1 sample with a volume fraction of $0.8 \%$ and an applied voltage of $400 \mathrm{mV}$ between the electrodes as an example. After the DC electric field was forced on the sample and the particles do not shift any more after hours of waiting, the reflection curves at different $x$ coordinates determined by a reflection spectrometer are shown in Figure 5, where the reflection curves adjacent to the electrodes have been omitted. It can be seen that the nearest interparticle distances $\left(d_{\text {exp }}\right)$ varied linearly along the $x$-axis. The relevant data points of $d_{\exp }$ along $x$ and their fitting lines are plotted in Figure 6. As can be seen from this figure, the variation trend of the nearest interparticle distances is rather uniform in the middle of the sample cell but not so good near the electrodes. The slop of the fitting line, $\mathrm{d}_{\exp }(x) / \mathrm{d} x=2.05 \times 10^{-7}$, was obtained through estimation of linear least squares. The 


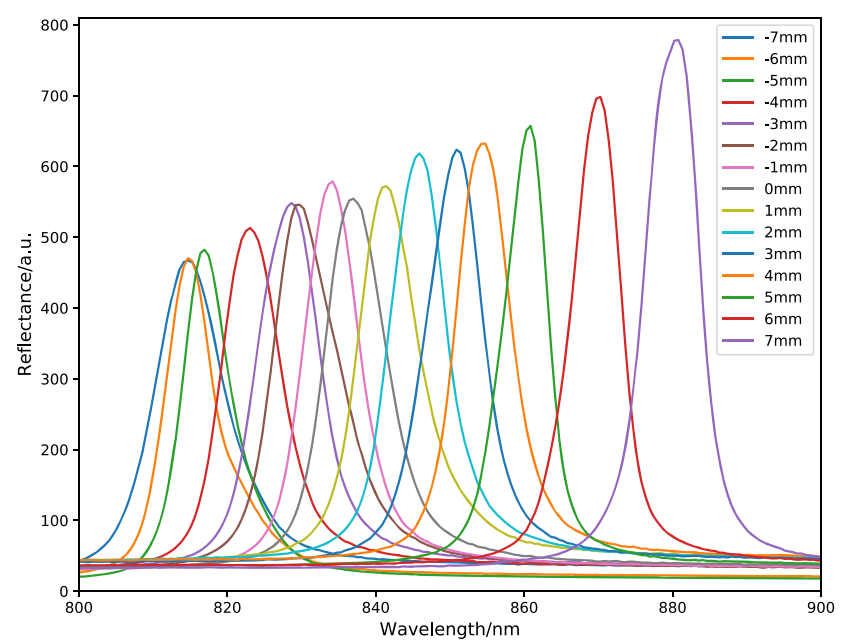

Figure 5. Reflection spectra of colloidal crystal at different $x$ coordinate (particle: PS- 1 , volume fraction: $0.8 \%$, electrode voltage: $0.4 \mathrm{~V})$.

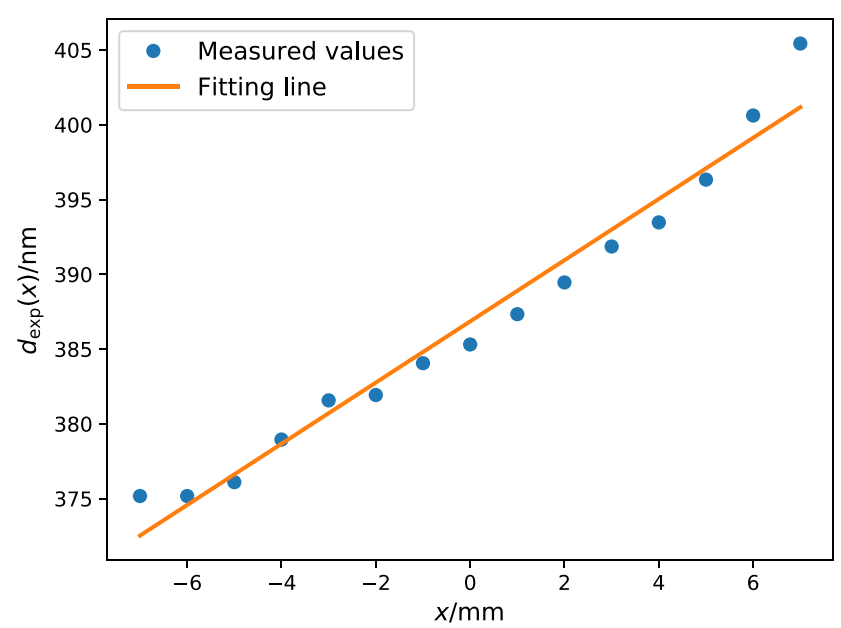

Figure 6. Nearest interparticle distances of the colloidal crystal. Slop of the fitting line: $d_{\exp }(x) / \mathrm{d} x=2.05 \times 10^{-7}$ (particle: PS-1; volume fraction: $0.8 \%$; electrode voltage: $0.4 \mathrm{~V}$ ).

voltage across R3 was also measured to be $0.473 \mathrm{mV}$. Therefore, by employing eq 29 above, the experimental value of the bulk modulus of the colloidal crystal with $0.8 \%$ particle volume fraction is determined as $18.2 \mathrm{~Pa}$, which agrees with the value of $19.3 \mathrm{~Pa}$ calculated according to eq 34 . For each particle volume fraction with different voltages $(400,500$, and $600 \mathrm{mV}$ ) applied, respectively, we made several measurements independently to get an averaged bulk modulus.

We have argued that $\varepsilon_{x x}, \varepsilon_{y y}$, and $\varepsilon_{z z}$ at one point should have the same value. To further confirm this judgment, we used the indium tin oxide (ITO) film coating on the inward surfaces of quartz cell sides normal to the $x$-axis to make the opaque platinum electrodes replaced by transparent ones. Since the two normal stresses $\varepsilon_{y y}$ and $\varepsilon_{z z}$ are perpendicular to the $x$-axis, they have equivalent value because of symmetry. Then, we just need to demonstrate that $\varepsilon_{x x}$ equals $\varepsilon_{y y}$, i.e., to demonstrate that the nearest interparticle distance of the colloidal crystal along the $x$-axis equals that along the $y$-axis at the same place. For one of the samples, the measured nearest interparticle distances along the $x$-axis is $248 \mathrm{~nm}$ at the place near the anode. The nearest interparticle distance along the $y$ - axis on the transparent ITO anode is $238 \mathrm{~nm}$. This value is close to $248 \mathrm{~nm}$ rather than $305 \mathrm{~nm}$, the average value of those along the $x$-axis or the value before the application of the electrical field. This confirmed that $\varepsilon_{y y}$ and $\varepsilon_{z z}$ are both equal to $\varepsilon_{x x}$

\section{RESULTS AND DISCUSSION}

This paper proposes two approaches, including an experimental method and a theoretical method, to determine the bulk modulus of the colloidal crystal formed by highly charged particles. The value through the experimental method is the measurement by exerting an electric field and that through the theoretical method is the reciprocal of compressibility due to the osmotic pressure of existing counterions.

As previously discussed, only when eq 17 is satisfied, eqs 29 and 34 become applicable. In other words, the prerequisite for eqs 29 and 34 to be valid is that the interparticle separation distance should be significantly greater than the individual particle diameter, namely, $\phi \ll 1$. Actually, to form crystals with $\phi \ll 1$, their constituent particles have to be highly charged. In this study, four different volume fractions were used for particles PS-1 and they were 0.6, 0.8, 1.0, and 1.2\%, respectively. Also, three different volume fractions were used for particles PS-2 and they were 1.2, 1.3, and $1.4 \%$, respectively. According to parameters listed in Table 1, it can be confirmed that the assumption for eq 17 is satisfied for both two kinds of colloidal particles in this study.

All of the theoretical and experimental results of the bulk moduli of colloidal crystals with different particle volume fractions are given in Table 2. From the table, we can find that

Table 2. Theoretical and Experimental Results of Bulk Moduli for Colloidal Crystals with Different Particle Volume Fractions

$\begin{array}{ccc}\phi(\%) & K_{\text {theoretical }} / \mathrm{Pa} & K_{\text {experimental }} / \mathrm{Pa} \\ 0.6 & \text { (a) PS-1 } & \\ 0.8 & 15.5 & 16.9 \pm 4.1 \\ 1.0 & 19.3 & 20.6 \pm 1.7 \\ 1.2 & 22.7 & 22.4 \pm 2.2 \\ & 27.5 & 28.4 \pm 2.7 \\ 1.2 & \text { (b) PS-2 } & \\ 1.3 & 41.4 & 40.9 \pm 2.5 \\ 1.4 & 43.8 & 42.3 \pm 2.3 \\ & 46.2 & 44.9 \pm 2.4\end{array}$

the experimental result is quite consistent with the theoretical one for each particle volume fraction. It also demonstrates that the higher the particle volume fraction, the bigger the bulk modulus. This variation trend is also shown in Figure 7.

Since the structure factor contains structural information of colloidal suspension, it also can be used to determine the bulk modulus, which is the reciprocal of compressibility. According to the previously reported formula of isothermal osmotic compressibility, the bulk modulus of the colloidal crystal ${ }^{18,20,57}$ can be evaluated by

$$
K_{\mathrm{S}}=\frac{n_{\mathrm{P}}}{\lim _{q \rightarrow 0} S(q)}
$$

where $n_{\mathrm{P}}$ is the number density of colloidal particles, $S$ is the static structure factor, and $q$ is the scattering wavenumber of the principal peak in $S(q)$. Gapinski et al. ${ }^{20}$ indicated that the 


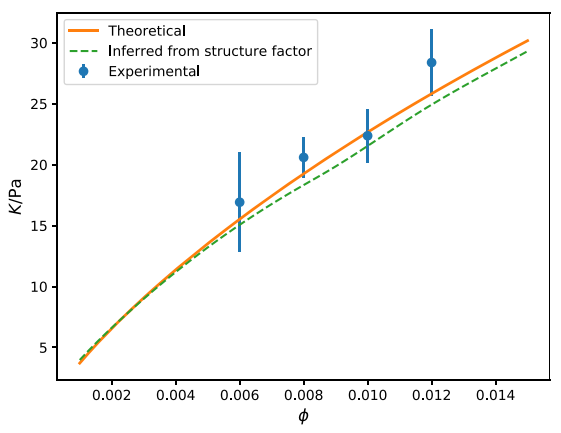

(a) PS-1

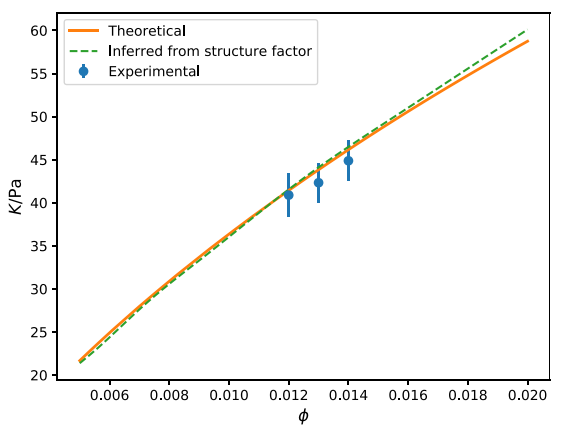

(b) PS-2

Figure 7. Theoretical and experimental values of the bulk moduli for different particle volume fractions.

zero limit static structure factor $\lim _{q \rightarrow 0} S(q)$ is only the function of $\lambda$, the reduced screening parameter of the colloidal suspension as shown in Figure 15 in their paper. By this function, we also give the lines of results using this calculation method in Figure 7. As mentioned above, the effective elasticity charge, $Z^{* \prime}$, is adopted to evaluate the reduced screening parameter $\lambda$.

Now, we have three results coming from three different approaches, respectively. The first is the experimental measurement by applying the DC electric field; the second is the evaluation of the theoretical model; and the third is the calculation by the aid of the static structure factor. Upon comparing the three results, we can see that they are quite consistent.

\section{CONCLUSIONS}

This paper first clarifies that neither the sedimentation equilibrium nor electrostatic field method is suitable for measuring Young's modulus of the colloidal crystal because the crystal will be unable to expand in other directions under unidirectional compression due to the container wall restraints. Then, we present an experimental scheme with corresponding theoretical formulation for measuring the bulk modulus by the electrostatic field method. Meanwhile, based on van't Hoff's law of osmotic pressure and the theory of Ohshima, a theoretical expression to independently predict the osmotic bulk modulus is also proposed. In our experiments to determine the bulk moduli by electrostatic field method, four different volume fractions $(0.6,0.8,1.0$, and $1.2 \%)$ were used for particles PS-1 and three different volume fractions (1.2, 1.3, and $1.4 \%$ ) were used for particles PS-2. The experimental results are in good agreement with those predicted by the proposed theoretical model as well as the calculation involving the zero-wavenumber static structure factor.
We hope that the result of this paper would be of certain significance for a better understanding of the elastic behavior of a colloidal crystal.

\section{AUTHOR INFORMATION}

\section{Corresponding Author}

*E-mail: xush@imech.ac.cn. Tel: +86-10-82544099.

ORCID $\odot$

Linwei Wang: 0000-0002-4544-8170

Shenghua Xu: 0000-0001-5433-0409

Notes

The authors declare no competing financial interest.

\section{ACKNOWLEDGMENTS}

We gratefully acknowledge financial support from the National Natural Science Foundation of China under grants 11672295 and 11572322 .

\section{REFERENCES}

(1) Pieranski, P. Colloidal Crystals. Contemp. Phys. 1983, 24, 25-73.

(2) Bosak, A.; Snigireva, I.; Napolskii, K. S.; Snigirev, A. HighResolution Transmission X-ray Microscopy: A New Tool for Mesoscopic Materials. Adv. Mater. 2010, 22, 3256-3259.

(3) Zhou, H.; Qin, Y.; Xu, S.; Sun, Z. Crystallization Kinetics of Concurrent Liquid-Metastable and Metastable-Stable Transitions, and Ostwald's Step Rule. Langmuir 2015, 31, 7204-7209.

(4) Sogami, I. S. On Adiabatic Pair Potentials of Highly Charged Colloid Particles. Prog. Theor. Exp. Phys. 2018, 2018, No. 033J01.

(5) Niu, R.; Palberg, T. Seedless Assembly of Colloidal Crystals by Inverted Micro-Fluidic Pumping. Soft Matter 2018, 14, 3435-3442.

(6) Okubo, T. Elasticity of the Crystal-Like, Amorphous Solid-Like, and Liquid-Like Structures in Deionized Suspensions of Colloidal Silica Spheres. Colloid Polym. Sci. 1993, 271, 873-883.

(7) Weiss, J. A.; Larsen, A. E.; Grier, D. G. Interactions, Dynamics, and Elasticity in Charge-Stabilized Colloidal Crystals. J. Chem. Phys. 1998, 109, 8659-8666.

(8) Ito, K.; Sumaru, K.; Ise, N. Elastic Properties of Colloidal Crystals. Phys. Rev. B 1992, 46, 3105-3107.

(9) Mundoor, H.; Senyuk, B.; Smalyukh, I. I. Triclinic Nematic Colloidal Crystals from Competing Elastic and Electrostatic Interactions. Science 2016, 352, 69-73.

(10) Mewis, J.; Wagner, N. J. Colloidal Suspension Rheology; Cambridge University Press: Cambridge, 2012.

(11) Arora, A. K.; Tata, B. Interactions, Structural Ordering and Phase Transitions in Colloidal Dispersions. Adv. Colloid Interface Sci. 1998, 78, 49-97.

(12) Crandall, R. S.; Williams, R. Gravitational Compression of Crystallized Suspensions of Polystyrene Spheres. Science 1977, 198, 293-295.

(13) Okubo, T. Elastic Modulus of Crystal-Like Structures of Deionized Colloidal Spheres in Sedimentation Equilibrium as Studied by the Reflection-Spectrum Method. J. Chem. Soc., Faraday Trans. 1 1989, 85, 455-466.

(14) Tomita, M.; van de Ven, T. G. M. Creating Color Patterns with Ordered Latices. J. Opt. Soc. Am. A 1984, 1, 317-318.

(15) Tomita, M.; de Ven, T. G. M. V. Electrically Induced Changes in Latex Structure. J. Phys. Chem. A 1985, 89, 1291-1296.

(16) Okubo, T. Time-Resolved Reflection Spectrum of Ordered Structure of Monodispersed Polystyrene Spheres in an Electric Field. J. Chem. Soc., Faraday Trans. 1 1987, 83, 2487-2495.

(17) Steytler, D. C.; Robinson, B. H.; Eastoe, J.; MacDonald, I.; Ibel, K.; Dore, J. C. Measurement of Inter-Particle Forces from the Osmotic Pressure of Partially Frozen Dispersions. J. Phys.: Condens. Matter 1996, 8, 9531-9536. 
(18) Junio, J.; Cohen, J. A.; Ou-Yang, H. D. Osmotic Bulk Modulus of Charged Colloids Measured by Ensemble Optical Trapping. J. Phys. Chem. B 2016, 120, 9187-9194.

(19) Gapinski, J.; Nägele, G.; Patkowski, A. Freezing Lines of Colloidal Yukawa Spheres. I. A Rogers-Young Integral Equation Study. J. Chem. Phys. 2012, 136, No. 024507.

(20) Gapinski, J.; Nägele, G.; Patkowski, A. Freezing Lines of Colloidal Yukawa Spheres. II. Local Structure and Characteristic Lengths. J. Chem. Phys. 2014, 141, No. 124505.

(21) Tan, B. H.; Tam, K. C.; Lam, Y. C.; Tan, C. B. Osmotic Compressibility of Soft Colloidal Systems. Langmuir 2005, 21, 42834290.

(22) Chen, B.-H.; Payandeh, B.; Robert, M. Turbidity and Critical Behavior of a Colloid-Polymer System. Phys. Rev. E 2001, 64, No. 042401.

(23) Apfel, U.; Grunder, R.; Ballauff, M. A Turbidity Study of Particle Interaction in Latex Suspensions. Colloid Polym. Sci. 1994, 272, 820-829.

(24) Grimson, M. J. Small-Angle Scattering from Colloidal Dispersions. J. Chem. Soc., Faraday Trans. 2 1983, 79, 817-832.

(25) Ibach, H.; Lüth, H. Solid-State Physics; Springer-Verlag: Heidelberg, 2009.

(26) Kesavamoorthy, R.; Arora, A. K. Gravitational Compression in Colloidal Suspension. J. Phys. A: Math. Gen. 1985, 18, 3389-3398.

(27) Timoshenko, S.; Goodier, J. N. Theory of Elasticity; 3rd ed.; Mcgraw-Hill: Chennai, 2010

(28) Landau, L. D.; Lifshitz, E. M. Theory of Elasticity, 3rd ed.; Elsevier Butterworth-Heinemann: Burlington, 1986.

(29) Alexander, S.; Chaikin, P. M.; Grant, P.; Morales, G. J.; Pincus, P.; Hone, D. Charge Renormalization, Osmotic Pressure, and Bulk Modulus of Colloidal Crystals: Theory. J. Chem. Phys. 1984, 80, $5776-5781$.

(30) Ashcroft, N. W. Solid State Physics; Thomson Press: New Delhi, 2003

(31) Carrique, F.; Arroyo, F.; Delgado, A. Electrokinetics of Concentrated Suspensions of Spherical Colloidal Particles with Surface Conductance, Arbitrary Zeta Potential, and Double-Layer Thickness in Static Electric Fields. J. Colloid Interface Sci. 2002, 252, 126-137.

(32) Ruiz-Reina, E.; Carrique, F. Electric Double Layer of Spherical Particles in Salt-Free Concentrated Suspensions: Water Dissociation and $\mathrm{CO}_{2}$ Influence. J. Phys. Chem. B 2008, 112, 11960-11967.

(33) Ohshima, H. Electrophoretic Mobility of Spherical Colloidal Particles in Concentrated Suspensions. J. Colloid Interface Sci. 1997, 188, 481-485.

(34) Hamann, C. H.; Hamnett, A.; Vielstich, W. Electrochemistry, 2nd ed.; Wiley-VCH: Weinheim, 2007.

(35) Rayleigh, L. XX. On the Equilibrium of Liquid Conducting Masses Charged with Electricity. Philos. Mag. 1882, 14, 184-186.

(36) Ohshima, H. Electrical Conductivity of a Concentrated Suspension of Spherical Colloidal Particles. J. Colloid Interface Sci. 1999, 212, 443-448.

(37) Serway, R. A.; Jewett, J. W. Physics for Scientists and Engineers; Cengage Learning: Boston, 2018.

(38) Carrique, F.; Arroyo, F.; Delgado, A. Electrokinetics of Concentrated Suspensions of Spherical Colloidal Particles: Effect of a Dynamic Stern Layer on Electrophoresis and DC Conductivity. J. Colloid Interface Sci. 2001, 243, 351-361.

(39) Atkins, P.; De Paula, J. Atkins' Physical Chemistry; 9th ed.; Oxford University Press: Oxford, 2010.

(40) Ohshima, H. Electrical Conductivity of a Concentrated Suspension of Soft Particles. J. Colloid Interface Sci. 2000, 229, 307-309.

(41) Ding, J. M.; Keh, H. J. The Electrophoretic Mobility and Electric Conductivity of a Concentrated Suspension of Colloidal Spheres with Arbitrary Double-Layer Thickness. J. Colloid Interface Sci. 2001, 236, 180-193.
(42) Ohshima, H. Surface Charge Density/Surface Potential Relationship for a Spherical Colloidal Particle in a Salt-Free Medium. J. Colloid Interface Sci. 2002, 247, 18-23.

(43) Ohshima, H. Electrophoresis of Colloidal Particles in a SaltFree Medium. Chem. Eng. Sci. 2006, 61, 2104-2107.

(44) Batchelor, G. K. An Introduction to Fluid Dynamics; Cambridge University Press: Cambridge, 2000.

(45) Jones, R. A. L. Soft Condensed Matter; Oxford University Press: Oxford, 2002.

(46) Hawkins, R. J.; Aldoroty, R. A. The Osmotic Bulk Modulus of a Colloid Crystal of Infinite Rod-Like Molecules. Chem. Phys. Lett. 1987, 137, 83-85.

(47) Schroeder, R.; Rudov, A. A.; Lyon, L. A.; Richtering, W.; Pich, A.; Potemkin, I. I. Electrostatic Interactions and Osmotic Pressure of Counterions Control the pH-Dependent Swelling and Collapse of Polyampholyte Microgels with Random Distribution of Ionizable Groups. Macromolecules 2015, 48, 5914-5927.

(48) Hessinger, D.; Evers, M.; Palberg, T. Independent Ion Migration in Suspensions of Strongly Interacting Charged Colloidal Spheres. Phys. Rev. E 2000, 61, 5493-5506.

(49) Gong, Y.-K.; Nakashima, K.; Xu, R. A Novel Method to Determine Effective Charge of Polystyrene Latex Particles in Aqueous Dispersion. Langmuir 2001, 17, 2889-2892.

(50) Palberg, T.; Kottal, J.; Bitzer, F.; Simon, R.; Worth, M.; Leiderer, P. Shear Modulus Titration in Crystalline Colloidal Suspensions. J. Colloid Interface Sci. 1995, 169, 85-89.

(51) Zhou, H.; Xu, S.; Sun, Z.; Zhu, R. Shear Moduli in Bcc-Fcc Structure Transition of Colloidal Crystals. J. Chem. Phys. 2015, 143, No. 144903.

(52) Wette, P.; Schöpe, H. J.; Palberg, T. Comparison of Colloidal Effective Charges from Different Experiments. J. Chem. Phys. 2002, 116, 10981-10988.

(53) Shapran, L.; Medebach, M.; Wette, P.; Palberg, T.; Schöpe, H.; Horbach, J.; Kreer, T.; Chatterji, A. Qualitative Characterisation of Effective Interactions of Charged Spheres on Different Levels of Organisation Using Alexandera's Renormalised Charge As Reference. Colloid Surf., A 2005, 270-271, 220-225.

(54) Wang, L.; Xu, S.; Zhou, H.; Sun, Z.; Xu, F. Effect of Void Structures in Crystalline Structure on the Shear Moduli of Charged Colloidal Crystals. Colloid Surf., A 2017, 516, 115-120.

(55) Du, X.; Xu, S.-H.; Sun, Z.-W.; Liu, L. Influence of Sedimentation on Crystallization of Charged Colloidal Particles. Chin. J. Chem. Phys. 2012, 25, 318-324.

(56) Okubo, T. Colloidal Crystallization As Compared with Polymer Crystallization. Polym. J. 2008, 40, 882-890.

(57) Nägele, G.; Klein, R.; Frisch, H. L. On Rheological Properties of Charge-Stabilized Spherical Colloids. Colloid Polym. Sci. 1988, 266, 437-440. 